\title{
Review Article \\ Endoscopic Optical Coherence Tomography (OCT): Advances in Gastrointestinal Imaging
}

\author{
Tejas S. Kirtane ${ }^{1}$ and Mihir S. Wagh ${ }^{2}$ \\ ${ }^{1}$ Department of Medicine, Washington Hospital Center, 110 Irving St NW, Washington, DC 20010, USA \\ ${ }^{2}$ Division of Gastroenterology, University of Florida, 1600 SW Archer Road, P.O. Box 100214, Gainesville, FL 32610, USA \\ Correspondence should be addressed to Mihir S. Wagh; mihir.wagh@medicine.ufl.edu
}

Received 27 September 2013; Accepted 21 December 2013; Published 26 February 2014

Academic Editor: Horia Stefãnescu

Copyright (c) 2014 T. S. Kirtane and M. S. Wagh. This is an open access article distributed under the Creative Commons Attribution License, which permits unrestricted use, distribution, and reproduction in any medium, provided the original work is properly cited.

In the rapidly evolving field of endoscopic gastrointestinal imaging, Optical Coherence Tomography (OCT) has found many diverse applications. We present the current status of OCT and its practical applications in imaging normal and abnormal mucosa in the esophagus, stomach, small and large intestines, and biliary and pancreatic ducts. We highlight technical aspects and principles of imaging, assess published data, and suggest future directions for OCT-guided evaluation and therapy.

\section{Introduction}

Endoluminal imaging in gastrointestinal endoscopy has seen the advent of rapidly evolving new modalities over the last decade. Narrow band imaging [1], confocal laser endomicroscopy [2], and Optical Coherence Tomography (OCT or VLE: Volumetric Laser Endomicroscopy) [3] are some of the newer imaging techniques that have shown promise in the early detection of dysplasia and mucosal cancers and surveillance of cancers after endoscopic therapy. We present a practical assessment of OCT and its clinical applications focusing on recent advances in OCT in the diagnosis and management of gastrointestinal diseases.

\section{OCT Technology}

Conceptually, OCT (or Volumetric Laser Endomicroscopy/VLE) is analogous to B-mode ultrasonography, with the exception that near infrared light in the 700 to $1500 \mathrm{~nm}$ range of wavelength is used instead of sound waves to generate an image of the mucosal structure and its abnormalities using an interferometer device setup. Initially described in 1999 [4], OCT has evolved over the years to allow for higher resolution and rapid imaging. Time domain OCT was described initially but could not achieve high scanning rates. Subsequently, Fourier domain OCT was developed which allows for rapid image acquisition and generation of real time in vivo 2dimensional and 3-dimensional mucosal renditions [5].

An OCT probe can be passed through the accessory channel of an endoscope and can be kept in contact with the mucosa of interest which allows for a resolution of 7-10 micrometers and an imaging depth of $2-3 \mathrm{~mm}$ depending on the wavelength of light used and the type of tissue being imaged $[6,7]$. This allows visualization of histologic morphology in real time, especially the epithelial structures such as villi, crypts, and squamous and intestinal epithelium. In depth technical details and principles of optics involved in OCT have been discussed extensively elsewhere $[8,9]$.

Some of the more recent commercially available or custom-made OCT probes are Nvision VLE Imaging System (Nine Point Medical, Cambridge, MA) and probes from Lightlab Imaging (Westford, MA).

\section{Gastrointestinal Applications}

In the gastrointestinal tract, OCT has been used for imaging of the esophagus, stomach, small and large intestine, and biliary and pancreatic ducts. However, much of the experience and practical utility with OCT has been with esophageal, biliary, and pancreatic duct imaging.

Salient in vivo studies in the human gastrointestinal tract using OCT are summarized in Table 1. 
TABLE 1: In vivo OCT studies in the human gastrointestinal tract.

\begin{tabular}{|c|c|c|c|}
\hline Year & Author & Number of patients & Anatomic location/pathology \\
\hline 1997 & A. M. Sergeev & 3 & Esophagus, stomach \\
\hline 2000 & B. E. Bouma & 32 & Barrett's esophagus \\
\hline 2000 & S. Jäckle & 22 & Esophagus, stomach, colon \\
\hline 2000 & M. V. Sivak Jr. & 72 & Esophagus, stomach, duodenum terminal ileum, colon, rectum \\
\hline 2000 & X. D. Li & 8 & Esophagus \\
\hline 2001 & U. Seitz & 4 & Bile ducts \\
\hline 2001 & J. M. Poneros & 121 & Barrett's esophagus \\
\hline 2001 & G. Zuccaro & 69 & Esophagus, stomach \\
\hline 2002 & J. M. Poneros & 5 & Bile ducts \\
\hline 2004 & B. Shen & 70 & Crohn's disease and ulcerative colitis \\
\hline 2005 & Isenberg & 33 & Barrett's esophagus \\
\hline 2005 & V.X. Yang & 22 & Esophagus, stomach, duodenum \\
\hline 2006 & J. A. Evans & 55 & High grade dysplasia/intramucosal carcinoma in Barrett's esophagus \\
\hline 2006 & P. A. Testoni & 15 & Pancreatic duct \\
\hline 2006 & E. Masci & 40 & Celiac disease \\
\hline 2007 & Y. Chen & 50 & Barrett's esophagus \\
\hline 2008 & P. Consolo & 35 & Ulcerative colitis and Crohn's disease \\
\hline 2009 & M. Arvanitakis & 37 & Biliary strictures \\
\hline 2009 & D. C. Adler & 4 & Colon, ulcerative colitis, radiation proctitis \\
\hline 2010 & W. Hatta & 62 & Superficial squamous cell esophageal cancer \\
\hline 2012 & T. H. Tsai & 13 & Barrett's esophagus \\
\hline 2012 & C. Zhou & 1 & Cervical inlet patch \\
\hline
\end{tabular}

\section{Esophageal Imaging with OCT}

OCT has been shown to demonstrate the five-layered esophageal wall with good correlation with histologic structures [10]. With newer advances in techniques for endoscopic mucosal resection (EMR) [11] and ablation (radiofrequency and cryotherapy), assessing the depth of invasion of mucosal cancers is vital, with a pivotal role for OCT. Indeed, studies have shown superiority of resolution for OCT compared to EUS specifically for visualization of the mucosa and submucosa [12].

OCT is of particular importance in imaging patients with Barrett's esophagus (BE). Patients with BE are at an increased risk for development of esophageal adenocarcinoma [13] and the incidence of esophageal adenocarcinoma has increased by $300-500 \%$ in white men in the last 30 years $[14,15]$.

The feasibility of OCT for carrying out in vivo real time imaging of Barrett's esophagus, high grade dysplasia and esophageal adenocarcinoma has been well demonstrated (Figures 1, 2, and 3). In their study using ultra-high resolution OCT, Chen and colleagues [16] demonstrated characteristic layered epithelium in a normal esophagus with normal architecture, while images of Barrett's esophagus corresponded to crypt-like glandular structures. High grade dysplasia and esophageal adenocarcinoma images exhibited more heterogeneous structures corresponding to irregular, heterogeneous tissue morphology from distorted and cribriform or villiform glandular architecture. A prospective study showed that OCT had a sensitivity of $68 \%$ and specificity of $82 \%$ with a diagnostic accuracy of $78 \%$ for detection of dysplasia in Barrett's esophagus [17]. In this study, Isenberg et al. used 314 pairs of OCT images and biopsy specimens from 33 patients and blinded four endoscopists and one pathologist to the histology results/real time OCT images and arrived at their findings using histology as a gold standard.

The current Seattle Protocol for surveillance for Barrett's esophagus recommends random 4 quadrant biopsies and leaves room for sampling error due to missed areas of dysplasia at random biopsies. OCT can be useful in guiding biopsies or eventually acquiring optical biopsies in lieu of standard biopsies. Each 3D-OCT data set provides approximately $160 \mathrm{~mm}^{2}$ ( $8 \mathrm{~mm}$ circumference $\times 20 \mathrm{~mm}$ pullback) coverage of the esophagus if the tissue is fully wrapped around the probe. This is approximately 30 to 60 times larger than the area sampled by jumbo biopsy forceps $\left(\sim 6 \mathrm{~mm}^{2}\right)$ and standard biopsy forceps $\left(\sim 2.5 \mathrm{~mm}^{2}\right)$ [18], thus, reducing sampling error.

OCT has also been used to show the surface morphology of rarer entities such as heterotopic gastric mucosa in the upper esophagus, also known as cervical inlet patch $[19,20]$.

An emerging utility of OCT can be in detecting Subsquamous Intestinal Metaplasia at the Gastroesophageal junction. Subsquamous Intestinal Metaplasia (SSIM) which has also been variably described as buried Barrett's glands or buried glands or subsquamous glands are areas of metaplastic columnar tissue present below normal appearing squamous mucosa (Figure 4). SSIM has been known to be present de novo $[21,22]$ in patients with $\mathrm{BE}$ and can persist in patients with $\mathrm{BE}$ after acid suppressive therapy and ablative therapies for Barrett's esophagus such as radiofrequency ablation, cryotherapy, or photodynamic therapy. Although the true 


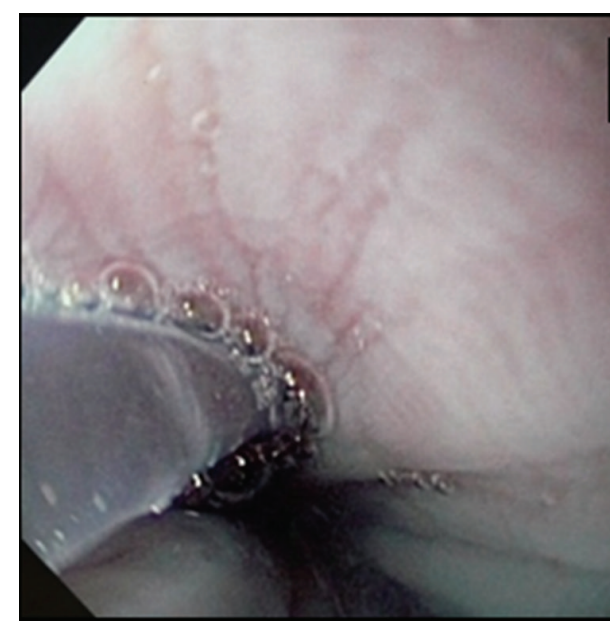

(a)

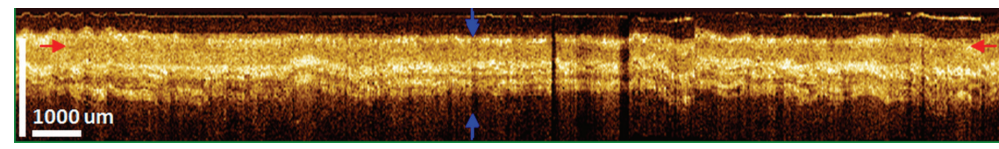

(b)

FIGURE 1: OCT imaging of normal esophagus. (a) Conventional endoscopy of the esophagus showing smooth pale mucosa. (b) Corresponding OCT image showing a well-defined, layered architecture. The epithelium, lamina propria, muscularis mucosa, submucosa, and muscularis propria are seen as distinct layers with alternating hypo- and hyperintensity.

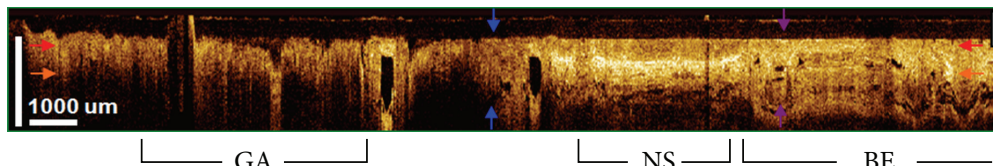

(a)

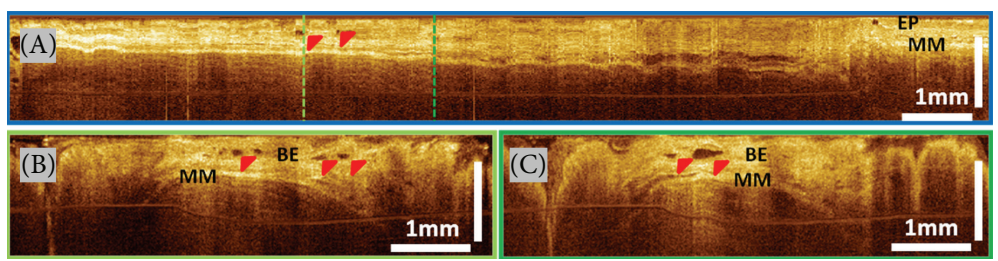

(b)

FIgURE 2: Barrett's esophagus (BE) without dysplasia. (a) Cross-sectional OCT imaging showing clear differences in layered architecture between gastric (GA), normal squamous (NS), and BE regions. BE regions exhibit distortion of the layered architecture and abnormal glandular features. (b) Cross-sectional OCT images around GEJ. BE glands (red arrows) are clearly observed (EP: epithelium; MM: muscularis mucosae in photos $\mathrm{A}-\mathrm{C})$.

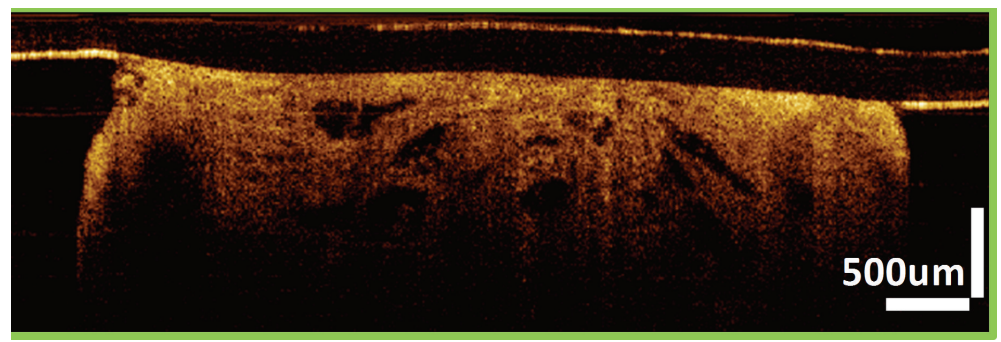

FIGURE 3: Intramucosal esophageal adenocarcinoma. OCT image showing dense large glands within the specimen. Lamina propria and muscularis mucosae (MM) layers are not clearly visible due to the infiltration of metaplasia into the MM layer. 


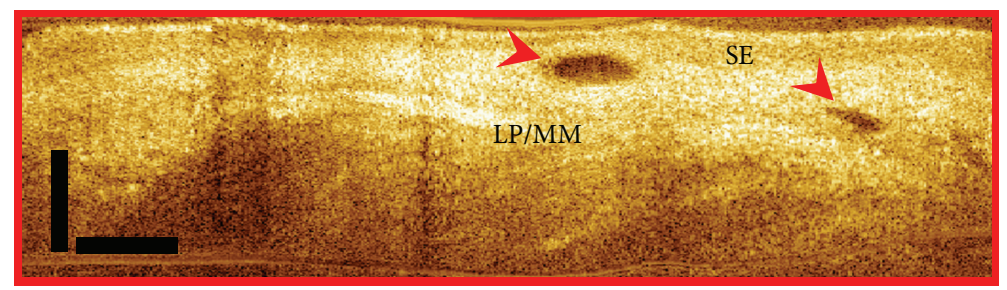

(a)

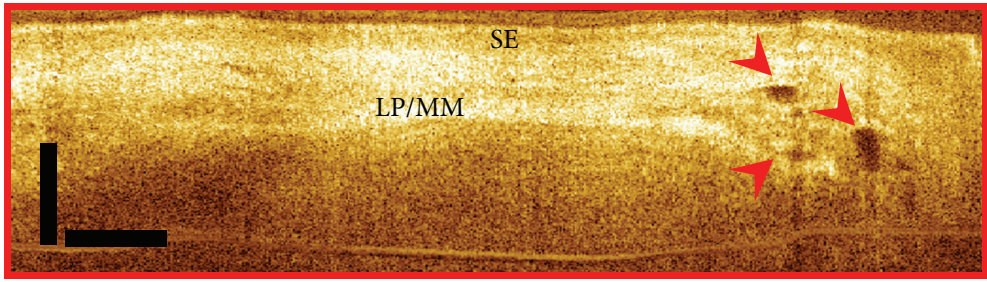

(b)

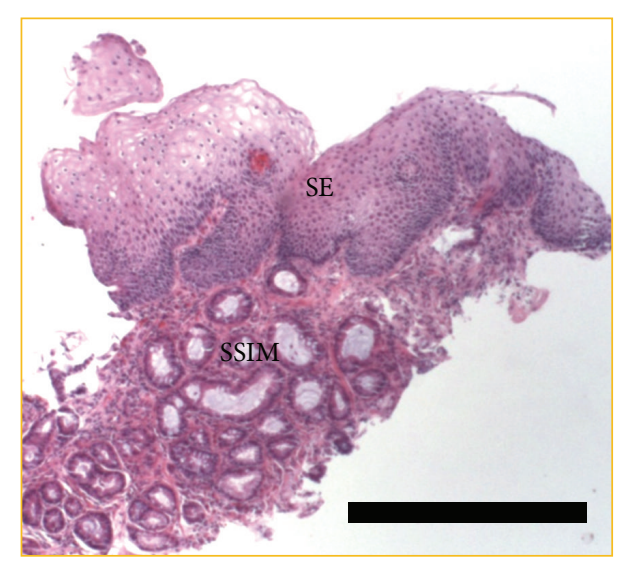

(c)

FIGURE 4: Subsquamous Intestinal Metaplasia (SSIM). (a-b): OCT images showing "buried glands" (red arrows). SE: squamous epithelium; LP/MM: lamina propria/muscularis mucosae. (c) Corresponding pathology showing subsquamous intestinal metaplasia under squamous epithelium.

malignant potential of residual SSIM is not known, concerns regarding identification and surveillance of SSIM are genuine owing to reports of progression to dysplasia and adenocarcinoma $[23,24]$. Also, SSIM evades detection on conventional white light and narrow band endoscopy and can be missed even on biopsy using standard forceps due to sampling error and insufficient depth as shown by Gupta et al. [25]. Recently, one group working with OCT has demonstrated the existence of subsquamous intestinal metaplasia after radiofrequency ablation of Barrett's esophagus using OCT technology $[18,26]$. This study showed regular flat squamous mucosa with small subepithelial vessels and glands in the normal esophagus. In contrast, BE showed large, densely packed glands with distortion of mucosal architecture. In post-RFA BE, findings were of a small number of isolated glands buried beneath 300-500 microns of neosquamous epithelium and lamina propria.

Going further, using OCT, it has been shown that the thickness of BE mucosa immediately after radiofrequency ablation predicts the response to RFA [27]. This study showed that BE mucosa was significantly thinner in patients who achieved complete eradication of intestinal metaplasia compared to those who did not achieve complete eradication of intestinal metaplasia at follow-up $(257 \pm 60 \mu \mathrm{m}$ versus $403 \pm$ $86 \mu \mathrm{m} ; P<0.0001)$. A threshold thickness of $333 \mu \mathrm{m}$ derived from receiver operating characteristic curves corresponded to a $92.3 \%$ sensitivity, $85 \%$ specificity, and $87.9 \%$ accuracy in predicting the presence of BE at follow-up. These findings may have important implications for the need for more RFA sessions.
OCT has also been used to delineate the difference in architectural changes after different endoluminal ablative therapies for Barrett's esophagus. Radiofrequency ablation was observed to induce 230 260 micrometer depth of architectural changes after each set of ablations over a particular region, while cryotherapy was observed to induce edema-like spongiform changes to $\sim 640 \mu \mathrm{m}$ depth [28].

\section{OCT in the Small Intestine}

There is limited data on the use of OCT in the small bowel. OCT has been used to image small intestinal mucosa and demonstrated $100 \%$ agreement with histology in a blinded study for differentiating between no atrophy and mild and marked atrophy of villous architecture [29]. This finding can be important to differentiate celiac disease from iron deficiency anemia in which villous architecture is typically preserved. An endoscopic doppler OCT has been used to show increased microvascularity in villi in duodenal adenomas [30].

\section{OCT in the Colon}

A number of studies have used OCT for evaluation of the large bowel. A study by Pfau et al. [31] showed that adenomas had significantly less structure and scattered light to a lesser degree than hyperplastic polyps and that hyperplastic polyps were significantly closer in organization and light scattering to normal mucosa as compared with adenomas. 
Other studies have characterized OCT findings in the normal colon, ulcerative colitis (UC), Crohn's disease (CD), and radiation proctitis [32-36]. The ability of OCT to image all the layers of the gastrointestinal wall can find utility in diagnosing the transmural inflammation of Crohn's disease (CD) and enable differentiating this from ulcerative colitis (UC). A prospective, blinded study by Shen and colleagues [37] showed a sensitivity of $90.0 \%$ and specificity of $83.3 \%$ for OCT in detecting the disrupted layered structure of the colon wall indicative of transmural inflammation, providing a valuable tool to distinguish CD from UC. This is especially relevant since biopsies are insufficient to assess for transmural inflammation.

\section{OCT in the Biliary and Pancreatic Ducts}

With the miniaturization of OCT probes, it is possible to use this technology for imaging the biliary and pancreatic ducts and evaluate strictures for neoplasia during ERCP. This was first demonstrated in vivo in the bile ducts by Seitz and colleagues in 2001 [38]. Their study demonstrated the layered architecture of the bile ducts similar to that found on histologic sections as well as underlying retroperitoneal structures with less backscattering. Similarly, OCT can recognize a differentiated three-layered architecture of the pancreatic duct in all cases with normal main pancreatic duct or chronic pancreatitis, whereas the layered architecture appears subverted in neoplastic lesions, with heterogeneous backscattering of the signal [39].

Given the low sensitivity (65\%) of brush cytology for detection of malignancy in biliary strictures even in combination with other sampling techniques such as biopsy forceps [40], OCT offers a promising alternative. OCT has indeed been shown to enhance the yield of brush cytology for detection of malignant biliary strictures. Arvanitakis and colleagues [41] showed that the diagnostic sensitivity for biliary strictures could be increased to $84 \%$ by combining biliary brushings with 2 OCT criteria which were a disorganized and subverted layer structure and large hypo- or nonreflective areas considered as tumor vessels.

Testoni and colleagues performed a prospective study in 12 patients using OCT imaging with ERCP [42]. Twelve consecutive patients with documented main pancreatic duct stricture were investigated by endoscopic ultrasonography (EUS) and ERCP, followed by brush cytology and OCT scanning. OCT recognized a differentiated three-layer architecture in all cases with normal main pancreatic duct or chronic pancreatitis, while in all the neoplastic lesions the layer architecture appeared totally subverted, with heterogeneous backscattering of the signal. The accuracy of OCT for detection of neoplastic tissue was $100 \%$ compared with $66.7 \%$ for brush cytology.

\section{Current Hurdles and Future Directions}

At present, different OCT probes differ in their scanning speed, resolution, and depth penetration. There is a an unmet need for establishment of uniform objective and subjective criteria which can be used by the endoscopist for real time assessment of mucosal characteristics which can aid in differentiating normal from neoplastic tissue and identify varying grades of dysplasia. While OCT can easily identify intestinal metaplasia within a normal esophagus, its ability to identify dysplasia within Barrett's esophagus is relatively poor as shown in a prospective study by Isenberg and colleagues [17] and it calls for further improvements in imaging technique, such as involving computer aided image analysis which can identify textures and patterns indicative of dysplasia which may be underappreciated by the human eye. Efforts are underway in using computer aided image analysis for detection of dysplasia in Barrett's esophagus [43]. A consensus on the various terminologies used for imaging technologies would help standardize methods and findings and avoid ambiguity. Comparison of OCT with other imaging technologies is needed, and, most importantly, larger prospective data assessing clinical outcomes with OCT imaging is crucial which can identify niche areas where OCT can be sensitive, reliable, and have a high impact with respect to determining further therapy for patients. There are limitations to every new technology and identifying specific high yield applications for OCT will be required before it can be routinely used by practicing gastroenterologists.

\section{Conclusions}

OCT is a promising noninvasive imaging technology easily accessible through the working channel of an endoscope. OCT imaging has been performed in various parts of the GI tract, though mainly restricted to major academic and research institutions. Limitations of OCT include relatively high costs, need for standardized terminology and criteria for normal and neoplastic tissues, and lack of prospective data on clinical outcomes. With further refinement of this technology, OCT may allow "true optical biopsies" in the future.

\section{Conflict of Interests}

The authors declare that there is no conflict of interests regarding the publication of this paper.

\section{Acknowledgments}

The authors are thankful to Hsiang-Chieh Lee from the Massachusetts Institute of Technology and Dr. Hiroshi Mashimo from the Boston VA Medical Center and Harvard Medical School for contributing figures used in this paper.

\section{References}

[1] L. M. Song, D. G. Adler, J. D. Conway et al., "Narrow band imaging and multiband imaging," Gastrointestinal Endoscopy, vol. 67, no. 4, pp. 581-589, 2008.

[2] H. Neumann, R. Kiesslich, M. B. Wallace, and M. F. Neurath, "Confocal laser endomicroscopy: technical advances and clinical applications," Gastroenterology, vol. 139, no. 2, pp. 388.e2392.e2, 2010. 
[3] G. J. Tearney, M. E. Brezinski, J. F. Southern, B. E. Bouma, S. A. Boppart, and J. G. Fujimoto, "Optical biopsy in human gastrointestinal tissue using optical coherence tomography," American Journal of Gastroenterology, vol. 92, no. 10, pp. 18001804, 1997.

[4] D. Huang, E. A. Swanson, C. P. Lin et al., "Optical coherence tomography," Science, vol. 254, no. 5035, pp. 1178-1181, 1991.

[5] D. C. Adler, C. Zhou, T. Tsai et al., "Three-dimensional endomicroscopy of the human colon using optical coherence tomography," Optics Express, vol. 17, no. 2, pp. 784-786, 2009.

[6] Y. Wang, J. S. Nelson, Z. Chen, B. J. Reiser, R. S. Chuck, and R. S. Windeler, "Optimal wavelength for ultrahigh-resolution optical coherence tomography," Optics Express, vol. 11, no. 12, pp. 14111417, 2003.

[7] M. E. Brezinski and J. G. Fujimoto, "Optical coherence tomography: high-resolution imaging in nontransparent tissue," IEEE Journal on Selected Topics in Quantum Electronics, vol. 5, no. 4, pp. 1185-1192, 1999.

[8] J. G. Fujimoto, C. Pitris, S. A. Boppart, and M. E. Brezinski, "Optical coherence tomography: an emerging technology for biomedical imaging and optical biopsy," Neoplasia, vol. 2, no. 1-2, pp. 9-25, 2000.

[9] J. G. Fujimoto, "Optical coherence tomography for ultrahigh resolution in vivo imaging," Nature Biotechnology, vol. 21, no. 11, pp. 1361-1367, 2003.

[10] A. M. Sergeev, V. M. Gelikonov, G. V. Gelikonov et al., "In vivo endoscopic OCT imaging of precancer and cancer states of human mucosa," Optics Express, vol. 1, no. 13, pp. 432-440, 1997.

[11] J. Ortiz-Fernandez-Sordo, A. Parra-Blanco, A. Garcia-Varona et al., "Endoscopic resection techniques and ablative therapies for Barrett's neoplasia," World Journal of Gastrointestinal Endoscopy, vol. 3, no. 9, pp. 171-182, 2011.

[12] A. Das, M. V. Sivak Jr., A. Chak et al., "High-resolution endoscopic imaging of the GI tract: a comparative study of optical coherence tomography versus high-frequency catheter probe EUS," Gastrointestinal Endoscopy, vol. 54, no. 2, pp. 219224, 2001.

[13] J.-F. Fléjou, "Barrett's oesophagus: from metaplasia to dysplasia and cancer," Gut, vol. 54, supplement 1, pp. i6-i12, 2005.

[14] S. S. Devesa, W. J. Blot, and J. F. Fraumeni Jr., "Changing patterns in the incidence of esophageal and gastric carcinoma in the United States," Cancer, vol. 83, no. 10, pp. 2049-2053, 1998.

[15] N. Shaheen and D. F. Ransohoff, "Gastroesophageal reflux, Barrett esophagus, and esophageal cancer: clinical applications," Journal of the American Medical Association, vol. 287, no. 15, pp. 1982-1986, 2002.

[16] Y. Chen, A. D. Aguirre, P.-L. Hsiung et al., "Ultrahigh resolution optical coherence tomography of Barrett's esophagus: preliminary descriptive clinical study correlating images with histology," Endoscopy, vol. 39, no. 7, pp. 599-605, 2007.

[17] G. Isenberg, M. V. Sivak Jr., A. Chak et al., "Accuracy of endoscopic optical coherence tomography in the detection of dysplasia in Barrett's esophagus: a prospective, double-blinded study," Gastrointestinal Endoscopy, vol. 62, no. 6, pp. 825-831, 2005.

[18] C. Zhou, T. Tsai, H. Lee et al., "Characterization of buried glands before and after radiofrequency ablation by using 3dimensional optical coherence tomography (with videos)," Gastrointestinal Endoscopy, vol. 76, no. 1, pp. 32-40, 2012.
[19] C. Zhou, T. Kirtane, T. Tsai et al., “Three-dimensional endoscopic optical coherence tomography imaging of cervical inlet patch," Gastrointestinal Endoscopy, vol. 75, no. 3, pp. 675-677, 2012.

[20] C. Zhou, T. Kirtane, T. H. Tsai et al., "Cervical inlet patchoptical coherence tomography imaging and clinical significance," World Journal of Gastroenterology, vol. 18, no. 20, pp. 2502-2510, 2012.

[21] J. Chennat, A. S. Ross, V. J. A. Konda et al., "Advanced pathology under squamous epithelium on initial EMR specimens in patients with Barrett's esophagus and high-grade dysplasia or intramucosal carcinoma: implications for surveillance and endotherapy management," Gastrointestinal Endoscopy, vol. 70, no. 3, pp. 417-421, 2009.

[22] M. Anders, Y. Lucks, M. A. El-Masry et al., "Subsquamous extension of intestinal metaplasia is detected in $98 \%$ of cases of neoplastic Barrett's esophagus," Clinical Gastroenterology and Hepatology, 2013.

[23] N. A. Gray, R. D. Odze, and S. J. Spechler, "Buried metaplasia after endoscopic ablation of Barrett's esophagus: a systematic review," American Journal of Gastroenterology, vol. 106, no. 11, pp. 1899-1909, 2011.

[24] M. Titi, A. Overhiser, O. Ulusarac et al., "Development of subsquamous high-grade dysplasia and adenocarcinoma after successful radiofrequency ablation of Barrett's esophagus," Gastroenterology, vol. 143, no. 3, pp. 564.el-566.el, 2012.

[25] N. Gupta, S. C. Mathur, J. A. Dumot et al., "Adequacy of esophageal squamous mucosa specimens obtained during endoscopy: are standard biopsies sufficient for postablation surveillance in Barrett's esophagus?" Gastrointestinal Endoscopy, vol. 75, no. 1, pp. 11-18, 2012.

[26] D. C. Adler, C. Zhou, T.-H. Tsai et al., "Three-dimensional optical coherence tomography of Barretts esophagus and buried glands beneath neosquamous epithelium following radiofrequency ablation," Endoscopy, vol. 41, no. 9, pp. 773-776, 2009.

[27] T. H. Tsai, C. Zhou, Y. K. Tao et al., "Structural markers observed with endoscopic 3-dimensional optical coherence tomography correlating with Barrett's esophagus radiofrequency ablation treatment response (with videos)," Gastrointestinal Endoscopy, vol. 76, no. 6, pp. 1104-1112, 2012.

[28] T. H. Tsai, C. Zhou, H. C. Lee et al., "Comparison of tissue architectural changes between radiofrequency ablation and cryospray ablation in Barrett's esophagus using endoscopic three-dimensional optical coherence tomography," Gastroenterology Research and Practice, vol. 2012, Article ID 684832, 8 pages, 2012.

[29] E. Masci, B. Mangiavillano, L. Albarello, A. Mariani, C. Doglioni, and P. A. Testoni, "Optical coherence tomography in the diagnosis of coeliac disease: a preliminary report," Gut, vol. 55 , no. 4, p. 579, 2006.

[30] V. X. D. Yang, S. Tang, M. L. Gordon et al., "Endoscopic Doppler optical coherence tomography in the human GI tract: initial experience," Gastrointestinal Endoscopy, vol. 61, no. 7, pp. 879890, 2005.

[31] P. R. Pfau, M. V. Sivak Jr., A. Chak et al., "Criteria for the diagnosis of dysplasia by endoscopic optical coherence tomography," Gastrointestinal Endoscopy, vol. 58, no. 2, pp. 196202, 2003.

[32] P. Consolo, G. Strangio, C. Luigiano, G. Giacobbe, S. Pallio, and L. Familiari, "Optical coherence tomography in inflammatory bowel disease: prospective evaluation of 35 patients," Diseases of the Colon and Rectum, vol. 51, no. 9, pp. 1374-1380, 2008. 
[33] L. Familiari, G. Strangio, P. Consolo et al., "Optical coherence tomography evaluation of ulcerative colitis: the patterns and the comparison with histology," American Journal of Gastroenterology, vol. 101, no. 12, pp. 2833-2840, 2006.

[34] B. Shen and G. Zuccaro Jr., "Optical coherence tomography in the gastrointestinal tract," Gastrointestinal Endoscopy Clinics of North America, vol. 14, no. 3, pp. 555-571, 2004.

[35] D. C. Adler, C. Zhou, T. Tsai et al., "Three-dimensional endomicroscopy of the human colon using optical coherence tomography," Optics Express, vol. 17, no. 2, pp. 784-796, 2009.

[36] C. Zhou, D. C. Adler, L. Becker et al., "Effective treatment of chronic radiation proctitis using radiofrequency ablation," Therapeutic Advances in Gastroenterology, vol. 2, no. 3, pp. 149156, 2009.

[37] B. Shen, G. Zuccaro Jr., T. L. Gramlich et al., "In vivo colonoscopic optical coherence tomography for transmural inflammation in inflammatory bowel disease," Clinical Gastroenterology and Hepatology, vol. 2, no. 12, pp. 1080-1087, 2004.

[38] U. Seitz, J. Freund, S. Jaeckle et al., "First in vivo optical coherence tomography in the human bile duct," Endoscopy, vol. 33, no. 12, pp. 1018-1021, 2001.

[39] P. A. Testoni and B. Mangiavillano, "Optical coherence tomography for bile and pancreatic duct imaging," Gastrointestinal Endoscopy Clinics of North America, vol. 19, no. 4, pp. 637-653, 2009.

[40] M. de Bellis, S. Sherman, E. L. Fogel et al., "Tissue sampling at ERCP in suspected malignant biliary strictures (Part 2)," Gastrointestinal Endoscopy, vol. 56, no. 5, pp. 720-730, 2002.

[41] M. Arvanitakis, L. Hookey, G. Tessier et al., "Intraductal optical coherence tomography during endoscopic retrograde cholangiopancreatography for investigation of biliary strictures," Endoscopy, vol. 41, no. 8, pp. 696-701, 2009.

[42] P. A. Testoni, A. Mariani, B. Mangiavillano, P. G. Arcidiacono, S. Di Pietro, and E. Masci, "Intraductal optical coherence tomography for investigating main pancreatic duct strictures," American Journal of Gastroenterology, vol. 102, no. 2, pp. 269274, 2007.

[43] X. Qi, Y. Pan, M. V. Sivak, J. E. Willis, G. Isenberg, and A. M. Rollins, "Image analysis for classification of dysplasia in Barrett's esophagus using endoscopic optical coherence tomography," Biomedical Optics Express, vol. 1, no. 3, pp. 825-847, 2010. 


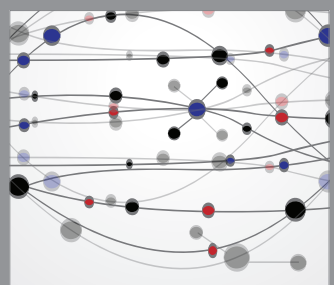

The Scientific World Journal
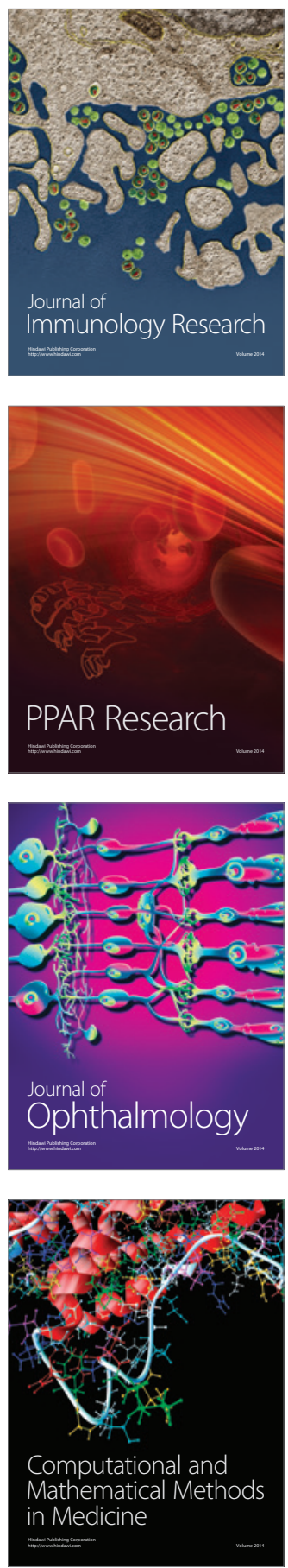

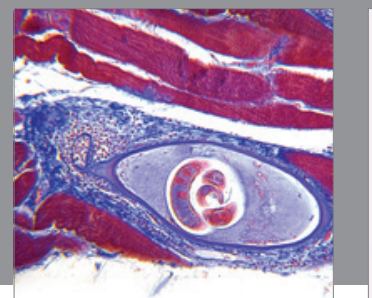

Gastroenterology

Research and Practice
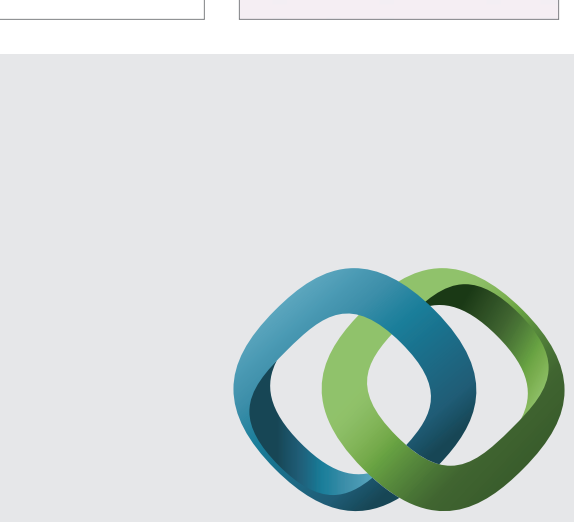

\section{Hindawi}

Submit your manuscripts at

http://www.hindawi.com
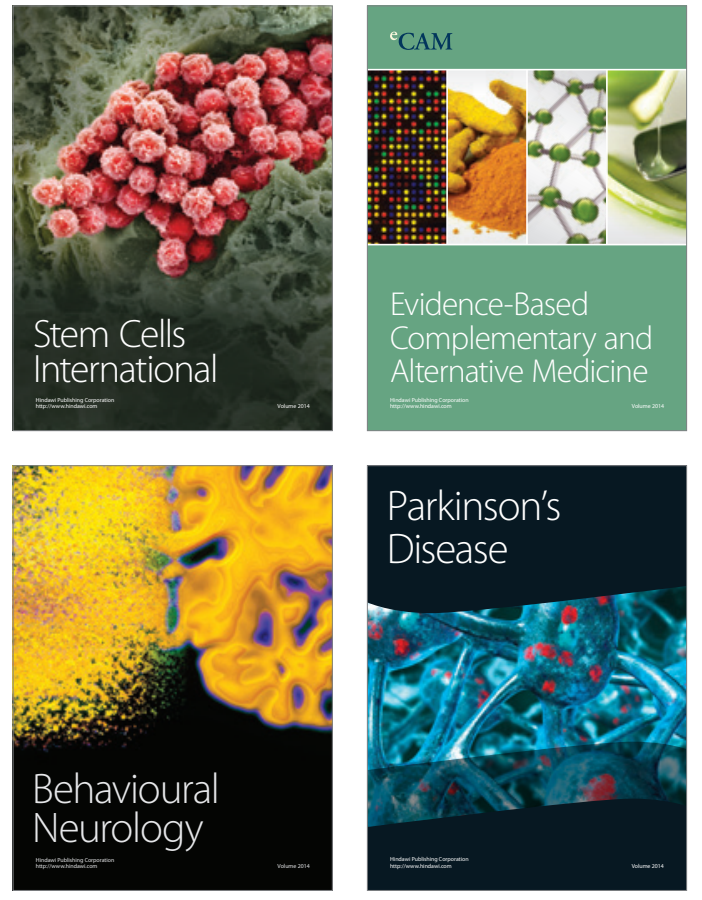
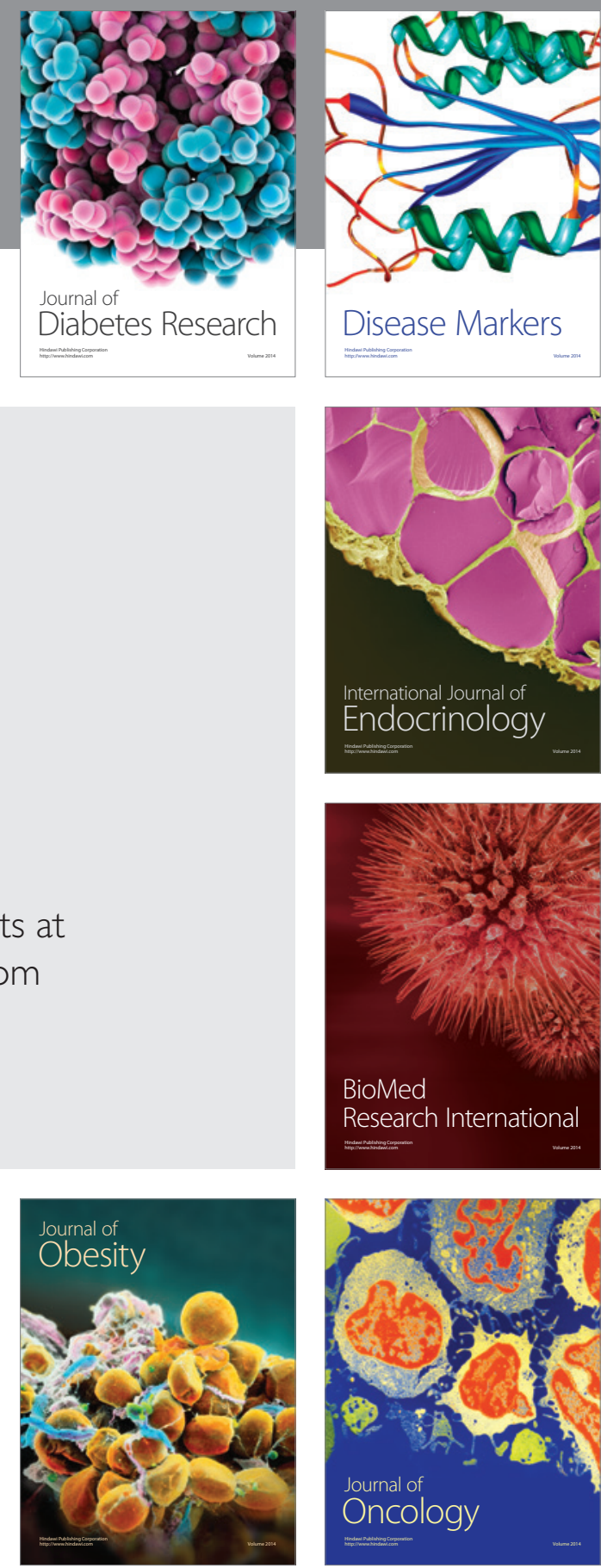

Disease Markers
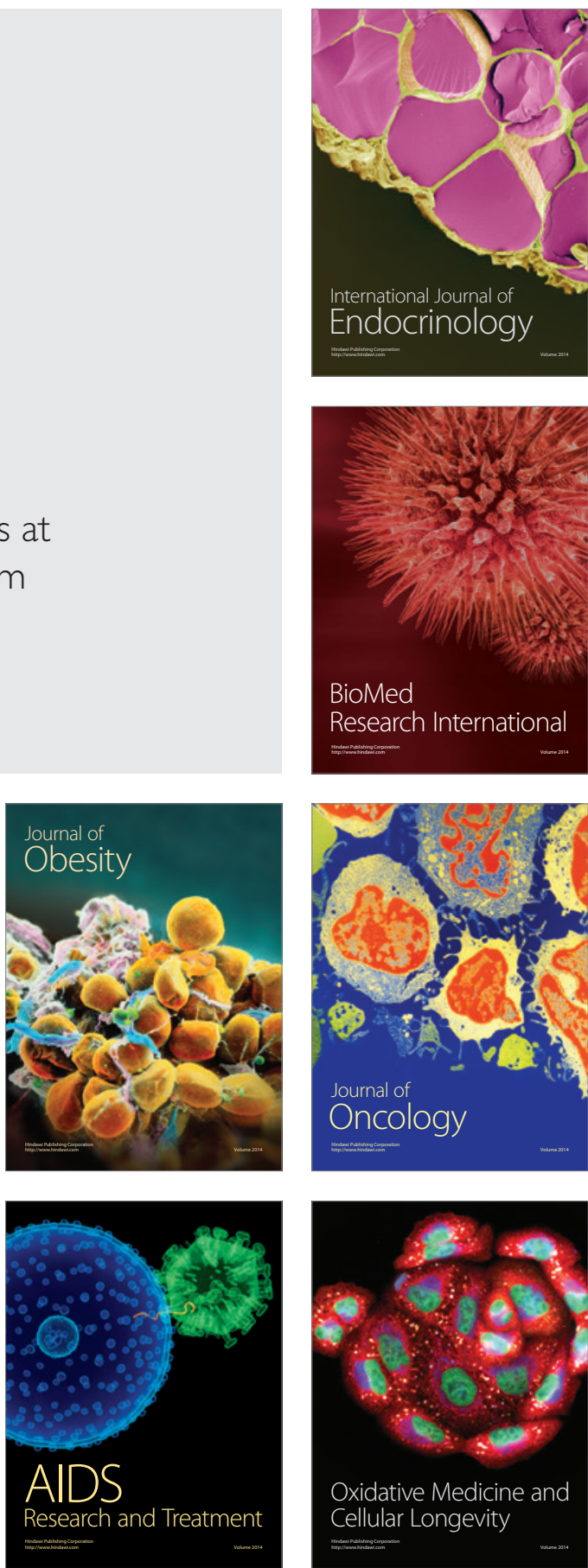